\title{
Drug shortages: Can we resolve that problem?
}

\author{
Mario Bedard, PharmD, RPh
}

Received: 6 March 2013/ Accepted: 14 March 2013/Published online: 20 March 2013

(c) Canadian Anesthesiologists' Society 2013

Although drug shortages have been an issue for several years, based on the unavailability of a number of drugs, 2012 has been the worst year for drug shortages in recent memory. The results of the national survey by Hall et al. published in this issue of the Journal highlight that fact. ${ }^{1}$ This increase in drug shortages is due in no small part to the unplanned partial closure of the Sandoz plant (Boucherville, QC, Canada) in early February 2012 and the downstream consequences of such a closure. It would be easy to blame Sandoz for all the problems relating to their poorly planned decision, but there are many factors that help explain why we are in such a difficult situation today.

Recent publications have summarized some of the causes of drug shortages, not only in Canada but also in the United-States and Europe. ${ }^{2,3}$ Issues regarding the availability of raw material, especially when obtained outside of North America, can cause disruptions in the manufacturing process. At the manufacturing level, there are problems such as surge capacity limitations, inspection failures, outdated equipment, and voluntary recalls for batches not meeting the standards of good manufacturing practices. When the American Food and Drug Agency tightens the regulatory requirements for drug manufacturing plants that distribute to the USA, such regulations affect Canada when the same plant produces drugs for our market. Business and market factors also affect the vulnerability of the system. Having more and more generic drugs produced by only one manufacturer (also called "sole source" drugs) in Canada decreases alternate options when the manufacturer faces a drug shortage. Large contracts with group purchasing

M. Bedard, PharmD, RPh $(\bowtie)$

Department of Pharmacy, The Ottawa Hospital, 501 Smyth

Road, Ottawa, ON K1H 8L6, Canada

e-mail: mbedard@toh.on.ca organizations (GPO) also reduce the incentive for other manufacturers to continue producing a drug that has not won an award with the GPO.

The Canadian government is also changing some of its regulatory requirements, which is slowing down the approval of manufacturing changes requested by drug companies. ${ }^{2}$ The result is a longer time needed to implement improvements and potentially being unable to meet current demand. There are many drugs on the lists of drug shortages in Canada and the USA. On February 24, 2013, the drug shortage section of the Web site for the American Society of Health-System Pharmacists (ASHP) listed 238 drugs that were affected by a shortage. ${ }^{4}$ On the same date, the Canadian Web site www.drugshortages.ca listed 282 formulations. ${ }^{5}$ These lists are getting longer every year, yet no clear strategy is in place to help reduce or eliminate them.

At the Drug Shortages Summit in November 2010, the American Society of Anesthesiologists, the American Society of Clinical Oncology, the ASHP, and the Institute for Safe Medication Practices met to discuss different aspects of the problem and to develop an action plan. The following top three drug shortage difficulties were identified: the absence of information on the duration of a specific shortage, the lack of advance warning of an impending problem, and the absence of information on the cause of the shortage. In Canada, the Sandoz partial closure has put pressure on the provincial and federal governments to improve our regulatory system relating to drug approval. It has also shone the spotlight on an issue that has been growing in recent years but, until recently, was not publicly known.

The survey by Hall et al. is significant for the fact that it measured, quite by chance, the impact of the Sandoz partial closure in the early days of the shortage crisis in February 
and March 2013. Their response rate (61.4\%) was impressive when compared with another recent survey completed on the same topic. ${ }^{6}$ Such a response rate strengthens the quality of the information gathered. We must also be cautious in interpreting answers that can be subjective in nature unless confirmed by objective evidence. Observations, such as rate of postoperative nausea and vomiting and longer stays in the postanesthesia care unit, may be biased by a few cases that tainted the overall perception regarding the shortages. It would be helpful to confirm the impact of shortages in postoperative patients using objective measures in a formal review.

The Canadian Medical Association, the Canadian Society of Hospital Pharmacists, and the Canadian Pharmacists Association have recently released some of the results of a survey that was sent to their members in October $2012 .^{6}$ The survey, completed by 1,070 members across Canada, provides insight into the problems associated with drug shortages in our country. Here are some of the results:

- $66 \%$ of physicians felt that shortages have worsened since 2010.

- $94 \%$ of pharmacists had trouble sourcing a drug in the last week.

- $76 \%$ of pharmacists indicated shortages have a significant impact on their workload.

- $64 \%$ of physicians felt that shortages had consequences for patients.

The groups jointly concluded that drug shortages have an impact on time available for patient care, and governments, industry, and other stakeholders need to work towards a system that eliminates or reduces the impact of drug shortages on patients and healthcare professionals.

Every hospital in Canada has been affected by the Sandoz shortage. The experience at The Ottawa Hospital (TOH) mirrors what was done elsewhere, but it is worth reviewing, if for no other reason than to improve next time. When Sandoz announced, without warning, that their production of injectable drugs was immediately being affected, TOH had to react quickly to implement an early action plan. Communication was a challenge, but the Pharmacy and Therapeutics (P\&T) Committee took the lead role within the institution. Meetings to review alternatives to the unavailable drugs were quickly scheduled with the heads of divisions and departments most affected by the upcoming shortages. Automatic substitutions were approved at the P\&T Committee and fast-tracked to the Medical Advisory Committee. The senior management team was also kept informed of the actions taken and approved extra resources as needed. All physicians, pharmacists, and nurses received daily then weekly e-mails summarizing the drug shortage status at TOH. The e-mails indicated which drugs were depleted or at critical low levels and also provided alternatives for each item identified. The hospital created a section on its intranet devoted to the communication of shortage information.

A regional group that included vice presidents, directors of pharmacy, communication specialists, and operation officers began meeting on a weekly basis to review critical lows and to collaborate on sharing expertise. The group, through their directors of pharmacy, also facilitated sharing drugs between hospitals when one of them was reaching critical lows. A representative from the regional group sat on a provincial committee that had been formed by the Ministry of Health to help deal with the acute situation. The Pharmacy Department worked internally to minimize wastage and started repackaging large multidose injectable vials into individual doses. This created a surge in workload but helped provide drugs to more patients. The Pharmacy Department also communicated widely to physicians and nurses when the drugs brought in had a different look or were packaged in a different concentration or volume than usual. Look-alike vials are a constant problem, but the risk was increased due to the fast rate of change and the number of changes that were happening concurrently. These measures helped avoid surgery cancellations and other negative patient impacts at $\mathrm{TOH}$.

Are there any solutions to resolve this shortage phenomenon? Some changes have begun to emerge since the 2012 crisis, but they are not yet broad enough to eliminate the problems in the supply chain that we have witnessed up to now. We have to realize that we cannot eliminate shortages completely since they are multifactorial in nature; however, the federal government needs to work with the provinces to set up a coordinated strategy to reduce some of the factors known to increase the risk of shortages. The federal government also needs to show leadership when shortages occur. They can facilitate access to drugs produced outside Canada, and they can reduce the paperwork that every hospital is required to complete in order to obtain access to these replacement drugs. Drug manufacturers should be mandated to provide an early warning when shortages are likely to occur and to provide more transparency when giving the reasons for the shortages. Health Canada must mandate that manufacturers provide a longer warning period when they decide to stop the production of a drug. This will provide more lead time for competitors to fill in the gap and ramp up production. Solesource drugs are putting patients at risk, and the Sandoz episode proved that point. Group purchasing organizations can play a major role in reducing the risk of creating solesource drugs by awarding large contracts to more than one manufacturer. This maintains competition and spreads production capacity. There is also the need for a drug information resource that can centrally assess and 
recommend alternatives when there is a drug shortage. This will avoid duplication of efforts within each hospital/ region/province.

The drug shortage of 2012 could have been worse, but we can't rely on our luck and assume future shortages will be less troublesome. Now is the time to work on a plan that will prevent the next shortage from becoming a disaster.

\section{Pénuries de médicaments: le problème peut-il être résolu?}

Bien que les pénuries de médicaments, définies comme la non-disponibilité de plusieurs médicaments, ne soient pas un problème nouveau, 2012 détient le record de la pire année dans le passé récent. Les résultats du sondage national de Hall et coll., publiés dans ce numéro du Journal, mettent en lumière ce problème. ${ }^{1}$ Cette augmentation des pénuries de médicaments est due en grande partie à la fermeture partielle imprévue de l'usine Sandoz (Boucherville, QC, Canada) au début du mois de février 2012 et des conséquences en aval d'une telle fermeture. Il serait facile d'accuser Sandoz de tous les problèmes découlant de leur décision mal planifiée, mais il existe de nombreux facteurs qui permettent d'expliquer pourquoi nous nous trouvons dans une situation si difficile aujourd'hui.

Plusieurs articles récents ont résumé certaines des raisons expliquant les pénuries de médicaments, non seulement au Canada mais également aux États-Unis et en Europe. $^{2,3}$ Les problèmes touchant à la disponibilité des matières premières, particulièrement lorsqu'elles proviennent d'ailleurs que l'Amérique du Nord, peuvent perturber le processus de fabrication. Au niveau de la fabrication, des problèmes peuvent être liés aux limites de la capacité d'un fabricant à intensifier sa production, aux manquements lors d'inspections, à un équipement désuet, et aux rappels volontaires de lots ne respectant pas les normes de bonnes pratiques de fabrication. Lorsque l'autorité de réglementation américaine, la FDA, resserre les exigences réglementaires pour les usines de fabrication de médicaments qui distribuent leurs produits aux États-Unis, si la même usine produit des médicaments pour notre marché, de tels règlements affecteront également le Canada. Les facteurs commerciaux ont également un impact sur la vulnérabilité du système. Le fait qu'un nombre croissant de médicaments génériques soient produits par un seul fabricant (également appelés médicaments à 'source unique') au Canada réduit les alternatives lorsqu'un fabricant est confronté à une pénurie. Les gros contrats avec les groupes d'achats (GPO) réduisent également la motivation d'autres fabricants à continuer de produire un médicament qui n'a pas été retenu par le GPO.

Le gouvernement canadien modifie également certaines de ses exigences réglementaires, ce qui ralentit le processus d'approbation des modifications de fabrication demandées par les compagnies pharmaceutiques. ${ }^{2}$ Il en découle qu'il faut davantage de temps pour mettre en œuvre ces améliorations et, possiblement, ne pas être en mesure de répondre à la demande actuelle. La liste des médicaments en pénurie au Canada et aux États-Unis est longue. Le 24 février 2013, la section concernant les pénuries de médicaments sur le site Internet de l'American Society of Health-System Pharmacists (ASHP) énumérait 238 médicaments affectés par une pénurie. ${ }^{4}$ À la même date, le site Internet de la Société canadienne des pharmaciens d'hôpitaux citait 282 produits. ${ }^{5}$ Ces listes s'allongent chaque année - et pourtant, aucune stratégie claire n'est en place pour contribuer à les réduire ou les éliminer.

Lors du Sommet américain sur les pénuries de médicaments en novembre 2010, l'American Society of Anesthesiologists, l'American Society of Clinical Oncology, l'ASHP, et l'Institute for Safe Medication Practices se sont rencontrés pour discuter de différentes facettes du problème et mettre au point un plan d'action. Les trois difficultés suivantes en matière de pénuries de médicaments ont été identifiées: l'absence d'informations sur la durée d'une pénurie spécifique, le manque d'avertissement préalable quant à un problème imminent, et l'absence d'informations sur la raison de la pénurie. Au Canada, la fermeture partielle de l'usine Sandoz a mis de la pression sur les gouvernements provinciaux et fédéral afin d'améliorer notre système de réglementation concernant l'approbation des médicaments. Cela a également mis en lumière un problème grandissant au cours des dernières années mais qui, jusqu'à récemment, n'était pas connu du public.

Le sondage de Hall et coll. est important en ce qu'il a mesuré, par hasard en fait, l'impact de la fermeture partielle de l'usine Sandoz durant les premiers jours de cette crise de pénurie en février et mars 2013. Leur taux de réponse $(61,4 \%)$ était impressionnant par rapport à un autre sondage récent réalisé sur le même thème. ${ }^{6}$ Un tel taux de réponse renforce la qualité des informations recueillies. Nous devons toutefois faire preuve de prudence dans l'interprétation de réponses, qui peuvent être subjectives de par leur nature, à moins qu'elles ne soient confirmées par des données probantes objectives. Certaines observations, telles que le taux de nausées et vomissements postopératoires et des durées prolongées en salle de réveil, pourraient être biaisées par quelques cas qui auront entaché la perception globale concernant les pénuries. Il serait utile de confirmer l'impact des pénuries chez les patients en période postopératoire à l'aide de mesures objectives dans un compte rendu formel. 
L'Association médicale canadienne, la Société canadienne des pharmaciens d'hôpitaux et l'Association des pharmaciens du Canada ont récemment publié certains des résultats d'un sondage envoyé à leurs membres en octobre $2012 .{ }^{6} \mathrm{Le}$ sondage, auquel ont répondu 1070 membres à travers le Canada, nous donne des pistes quant aux problèmes associés aux pénuries de médicaments dans notre pays. Voici quelques-uns de ces résultats:

- $66 \%$ des médecins pensent que les pénuries se sont empirées depuis 2010.

- $94 \%$ des pharmaciens ont eu du mal à s'approvisionner pour un médicament au cours de la semaine précédente.

- $76 \%$ des pharmaciens ont indiqué que les pénuries avaient un impact significatif sur leur charge de travail.

- $64 \%$ des médecins pensent que les pénuries ont des conséquences pour les patients.

Les groupes ont conjointement conclu que les pénuries de médicaments ont un impact sur le temps disponible pour les soins aux patients et que les gouvernements, l'industrie et les autres acteurs ont besoin de travailler à la conception d'un système qui éliminerait ou réduirait l'impact des pénuries de médicaments sur les patients et les professionnels de la santé.

Tous les hôpitaux canadiens ont été affectés par la pénurie liée à la fermeture partielle de l'usine Sandoz. L'expérience à l'Hôpital d'Ottawa (HO) reflète ce qui a été fait ailleurs, mais il est intéressant de la passer en revue, si ce n'est pour faire mieux la prochaine fois. Lorsque Sandoz a annoncé, sans préavis, que leur production de médicaments injectables était immédiatement affectée, l'HO a dû réagir rapidement afin de mettre en œuvre un plan d'action précoce. La communication s'est avérée être un défi, mais le Comité de pharmacologie et de thérapeutique (P\&T) a pris la tête de ce dossier au sein de l'institution. Des réunions ont été rapidement planifiées avec les responsables des divisions et des départements les plus affectés par les pénuries imminentes pour passer en revue les alternatives aux médicaments indisponibles. Des substitutions automatiques ont été approuvées par le Comité de P\&T et traitées en priorité par le Comité consultatif médical. L'équipe de la haute direction a également été tenue informée des mesures prises et a approuvé l'allocation de ressources supplémentaires selon le besoin. Tous les médecins, pharmaciens et infirmières ont reçu des courriels quotidiens puis hebdomadaires résumant l'état de la pénurie de médicaments à l'HO. Les courriels indiquaient quels médicaments étaient épuisés ou à des niveaux dangereusement bas et proposaient des alternatives à tous les produits identifiés. L'hôpital a créé une section sur son intranet dédiée à la communication des informations concernant les pénuries.

Un groupe régional incluant des vice-présidents, des directeurs de départements de pharmacie, des spécialistes de la communication et des directeurs d'exploitation, a commencé à se rencontrer sur une base hebdomadaire pour passer en revue les stocks dangereusement bas et pour collaborer en partageant leur expertise. Le groupe, par l'entremise des directeurs des départements de pharmacie, a également facilité le partage des médicaments entre les hôpitaux lorsque l'un venait à en manquer dangereusement. Un représentant du groupe régional a pris part à un comité provincial formé par le ministère de la Santé afin d'aider à gérer cette situation de crise. Le département de pharmacie a travaillé à l'interne afin de minimiser le gaspillage et a commencé à diviser les grands flacons injectables à doses multiples en doses individuelles. Cela a créé une augmentation de la charge de travail mais a permis d'offrir des médicaments à un nombre plus élevé de patients. Le département de pharmacie a également communiqué avec les médecins et les infirmières lorsque les médicaments reçus avaient une apparence différente ou étaient présentés dans une concentration différente ou un volume différent qu'à l'accoutumée. Les fioles qui se ressemblent sont un problème constant, mais le risque était d'autant plus grand en raison de la rapidité du changement et du nombre de changements survenant simultanément. Ces mesures ont contribué à éviter l'annulation de chirurgies et d'autres impacts négatifs sur les patients à l'HO.

Y a-t-il une solution pour résoudre ce phénomène de pénurie? Certains changements ont commencé à émerger depuis la crise de 2012, mais ils ne sont pas encore assez répandus pour éliminer les problèmes dans la chaîne d'approvisionnement dont nous avons été témoins jusqu'à présent. Nous devons désormais réaliser que nous ne pouvons éliminer complètement les pénuries, celles-ci étant multifactorielles; toutefois, le gouvernement fédéral doit travailler avec les provinces afin de mettre en place une stratégie coordonnée pour réduire certains des facteurs connus comme augmentant le risque de pénurie. Le gouvernement fédéral doit également faire preuve de leadership lorsqu'il y a pénurie. Il peut faciliter l'accès aux médicaments produits hors Canada, et il peut réduire la paperasse que chaque hôpital doit remplir afin d'avoir accès à ces médicaments de substitution. Les fabricants de médicaments devraient être tenus de fournir un préavis lorsqu'une pénurie est probable et d'être plus transparents dans la communication des raisons de cette pénurie. Santé Canada doit exiger des fabricants qu'ils l'avertissent plus tôt lorsqu'ils décident de cesser la production d'un médicament. Ainsi, les compétiteurs auront davantage de temps pour combler le fossé et accélérer la production. Les médicaments à source unique mettent les patients en danger, l'affaire Sandoz l'a prouvé. Les groupes d'achats peuvent jouer un rôle crucial pour réduire le risque que des médicaments à source unique soient créés en attribuant les gros contrats à plusieurs fabricants. Ainsi, on maintient la compétition et on répartit la capacité de production. Il faut 
également que soit créé un organisme mettant à disposition des informations sur les médicaments, lequel pourra évaluer de façon centrale les pénuries et recommander, au besoin, des alternatives. Ceci permettra d'éviter la duplication des efforts au sein de chaque hôpital/région/province.

La pénurie de médicaments de 2012 aurait pu être pire, mais nous ne pouvons compter sur notre bonne fortune et prendre pour acquis que les pénuries futures seront moins problématiques. C'est maintenant le bon moment pour travailler à un plan qui permettra d'éviter que la prochaine pénurie ne soit catastrophique.

Competing interests None declared.

Conflits d'intérêt Aucun.

\section{References}

1. Hall R, Bryson GL, Flowerdew, et al. Drug shortages in Canadian anesthesia: a national survey. Can J Anesth 2013; 60: this issue.
2. Morrison A. Drug Supply Disruptions (Environmental Scan Issue 17). Ottawa: Canadian Agency for Drugs and Technologies in Health; 2011. Available from URL: http://www.cadth.ca/en/ products/environmental-scanning/environmental-scans/environmen tal-scans-18 (accessed March 2013).

3. American Society of Health-Systems Pharmacists. Drug Shortages Summit. Bethesda: 2010. Available from URL: www.ashp.org/ drugshortages/summitreport (accessed March 2013).

4. American Society of Health-Systems Pharmacists. Bethesda (MD): American Society of Health-Systems Pharmacists; c2013. Drug Shortages: Current Drugs. Current Drug Shortage Bulletins; 2013. Available from URL: www.ashp.org/DrugShortages/Current (accessed March 2013).

5. Canadian Drug Shortage Database. Drug Shortages, 2013. Available from URL: www.drugshortages.ca (accessed March 2013).

6. Canadian Society of Hospital Pharmacists. CSHP Speaks Up on Drug Shortages. Ottawa: January 14, 2013. Available from URL: www.cshp.ca/advocacy/CSHPspeaks/drugShortages_e.asp (accessed March 2013). 